\title{
Barreras en la atención de la morbilidad materna en Yaxkukul, Yucatán, México
}

\section{Barriers in maternal morbidity care in Yaxkukul, Yucatan, Mexico}

RODRÍGUEZ-ANGULO, Elsa†* SOLÍS-RIVERO, Marita, OJEDA-RODRÍGUEZ, Ricardo y ANDUEZA-PECH, Guadalupe

Universidad Autónoma de Yucatán, “Centro de Investigaciones Regionales "Dr. Hideyo Noguchi”.

ID $1^{\mathrm{er}}$ Autor: Elsa, Rodríguez-Angulo / ORC ID: 0000-0001-5959-1341, Researcher ID Thomson: S-4625-2018, CVU CONACYT ID: 111408 ID $1{ }^{\text {er }}$ Coautor: Marita, Solís-Rivero / ORC ID: 0000-0002-1521-4853, ID Thomson: X-2749-2019, CVU CONACYT ID:
1009600

ID $2^{\text {do }}$ Coautor: Ricardo, Ojeda-Rodríguez / ORC ID: 0000-0002-2556-5847, Researcher ID Thomson: S-4761-2018, CVU CONACYT ID: 946773

ID $3^{\text {er }}$ Coautor: Guadalupe Andueza-Pech / ORC ID: 0000-0002-3083-0529, Researcher ID Thomson: S-4702-2018, CVU CONACYT ID: 84374

DOI: $10.35429 / \mathrm{JOHS} .2019 .18 .6 .28 .37$

Recibido Enero 11, 2019; Aceptado Marzo 23, 2019

\begin{abstract}
Resumen
Objetivos. Identificar las barreras que causan demoras en la ruta de atención obstétrica en mujeres embarazadas de Yaxkukul, Yucatán, de enero de 2016 a mayo de 2018. Metodología. Estudio observacional, descriptivo, transversal y retrospectivo. Se entrevistaron embarazadas que acudieron a control prenatal al centro de salud rural de Yaxkukul y se revisaron sus expedientes clínicos. Se estudiaron características sociodemográficas, control prenatal y se describió la ruta crítica de atención obstétrica, bajo el modelo de las tres demoras. Se calcularon porcentajes, medidas de tendencia central y dispersión; así como chi cuadrada para buscar asociación entre demoras y morbilidad materna. Se utilizó un nivel de confianza del $95 \%$ y un valor de $\mathrm{p}<0.05$. Contribución. El presente estudio contribuye a la prevención de la mortalidad materna y perinatal. Conocer las barreras que causan demoras en la atención permite identificar deficiencias en el protocolo de emergencias obstétricas establecidos en las unidades de salud rurales, para mejorar la calidad de la atención obstétrica.
\end{abstract}

Barreras, Demoras en la atención, Mujeres embarazadas

\begin{abstract}
Objectives. Identify the barriers that cause delays in the route of obstetric care in pregnant women of Yaxkukul, Yucatán, from January 2016 to May 2018. Methodology. Observational, descriptive, cross-sectional and retrospective study. Pregnant women who attended prenatal control at the rural health center of Yaxkukul were interviewed and reviewed their clinical record. Sociodemographic characteristics, prenatal control were studied and the critical route of obstetric care was described, under the model of the three delays. Percentages, measures of central tendency and dispersion were calculated; as well as square chi to look for association between delays and maternal morbidity. A $95 \%$ confidence level and a value of $\mathrm{p}<0.05$ were used. Contribution. The present study contributes to the prevention of maternal and perinatal mortality. Knowing the barriers that cause delays in care can identify deficiencies in the obstetric emergency protocol established in rural health units, to improve the quality of obstetric care.
\end{abstract}

Barriers, Delays in care, Pregnant women

Citación: RODRÍGUEZ-ANGULO, Elsa, SOLÍS-RIVERO, Marita, OJEDA-RODRÍGUEZ, Ricardo y ANDUEZA-PECH, Guadalupe. Barreras en la atención de la morbilidad materna en Yaxkukul, Yucatán, México. Revista de Ciencias de la Salud. 2019. 6-18: 28-34.

\footnotetext{
*Correspondencia al Autor (Correo Electrónico: rangulo@ correo.uady.mx)

$\dagger$ Investigador contribuyendo como primer Autor
} 


\section{Introducción}

La mortalidad materna (MM) es un problema multifactorial de salud pública que ha motivado a las regiones del mundo a reaccionar ante esta tragedia. A pesar de los esfuerzos realizados por instituciones internacionales no se lograron alcanzar las metas descritas en la Declaración de Alma Ata en septiembre de 1978, ni las planteadas en las metas del milenio para lograr la reducción de la mortalidad materna en un $75 \%$ de los niveles de 1990 a cumplirse en el año 2015.

La MM está estrechamente ligada a los estándares de la atención obstétrica. Thaddeus y Maine en 1994, reportaron en un estudio de revisión que muchas mujeres embarazadas llegan a los establecimientos de salud en estado de gravedad lo que empeora su pronóstico, y que el tiempo que se tarda en proporcionarles los cuidados necesarios es el factor crucial en su fallecimiento. A partir de estas observaciones se desarrolló el "modelo de las tres demoras".

En el mundo, cada día mueren alrededor de 830 mujeres por complicaciones relacionadas con el embarazo o el parto. En el 2015 se estimaron unas 303000 muertes de mujeres durante el embarazo y el parto o después de ellos. Prácticamente todas estas muertes se producen en países de ingresos bajos y la mayoría de ellas podrían haberse evitado.

La MM se define como la muerte de una mujer durante el embarazo, parto o puerperio por causas relacionadas o agravadas por el embarazo, pero no por razones accidentales. De acuerdo con la Organización Mundial de la Salud (OMS), la MM es "el fallecimiento de una mujer mientras está embarazada o dentro de los 42 días después de la terminación del embarazo, independientemente de la duración y lugar del mismo, producida por cualquier causa relacionada o agravada por el embarazo o su manejo, pero no por causas accidentales o incidentales.

Las principales causas de muerte para la mujer embarazada son: enfermedad hipertensiva inducida por embarazo (20.4\%), hemorragia obstétrica (19\%) y sepsis (4.1\%). Estos porcentajes son 5 a 10 veces superiores a los que se registran en los países industrializados o con mayor desarrollo de sus sistemas nacionales de salud.
Desde las primeras evidencias de demoras o retrasos descritas por Tahddeus y Main en 1993, se han realizado trabajos que las analizan a través de un modelo basado en la forma en que la mujer, su familia y los servicios de salud responden a la complicación. Las demoras han sido agrupadas en tres:

Demora 1, retraso en decidir buscar atención; depende de las capacidades y oportunidades de la mujer y su entorno para reconocer una complicación que amenaza la vida, así como de la información de la que dispone acerca de a dónde puede acudir.

Demora 2, retraso en identificar y acceder a un servicio de salud; depende de la distancia al servicio de salud, la disponibilidad y eficiencia del transporte y el costo de los traslados.

Demora 3, en obtener el tratamiento adecuado y oportuno; depende de la disponibilidad de personal capacitado con la que cuenta la institución, la disponibilidad de medicamentos e insumos (antibióticos, sulfato de magnesio, sangre segura) y de la infraestructura (quirófanos) del servicio de salud. En México, hay estudios que han analizado las demoras en el proceso de atención de la embarazada y de acuerdo con sus resultados recomiendan realizar intervenciones dirigidas a las etapas tempranas de la complicación y a disminuir las diversas formas de inequidad de género y socioeconómicas. Cualquier demora en recibir el tratamiento apropiado en mujeres embarazadas puede costarle su salud.

En Yucatán, existen evidencias de que, en zonas muy marginadas, la hemorragia es la causa principal de muerte materna. Las defunciones causadas por complicaciones obstétricas se relacionan con la forma en que las mujeres responden a las señales de alarma que indican una complicación y con la respuesta de los servicios de salud para el cuidado y el tratamiento oportuno de ésta. Esto es, las muertes maternas no son accidentales, sino que obedecen a un conjunto de factores que, al enlazarse, forman una cadena fatal de demoras o retrasos que impiden prevenir riesgos durante los embarazos, limitan el acceso a servicios de salud de calidad y traen como consecuencia la falta de atención oportuna de la complicación y, por consiguiente, la muerte. 


\section{Objetivo General}

Identificar las barreras que causan demoras en la ruta de atención obstétrica en mujeres embarazadas de Yaxkukul, Yucatán, de enero de 2016 a mayo de 2018.

\section{Material y Métodos}

El tipo de estudio fue observacional, descriptivo, transversal y retrospectivo. Se incluyeron mujeres embarazadas de la población del municipio de Yaxkukul y comisarias pertenecientes a dicha cabecera: Nolo y Tixkuncheil; que acudieron a control prenatal en el centro de salud rural de Yaxkukul, Yucatán durante el periodo de enero de 2016 a mayo de 2018. Se realizaron entrevistas durante la consulta prenatal y en los domicilios de las embarazadas. Previo consentimiento informado

\section{Definición operacional de variables.}

Edad. Tiempo cronológico de vida cumplido por el paciente al momento de la entrevista.

Escolaridad. Grado máximo de estudio alcanzado por la paciente al momento de la entrevista.

Ocupación. Tipo de trabajo que desempeña la paciente, con o sin remuneración económica.

Método de planificación familiar. Método de anticoncepción utilizado a lo largo de su vida fértil referido por la paciente.

Control prenatal. Numero de asistencia a citas de control prenatal a lo largo del último embarazo.

Primera demora (barreras). Demora en decidir buscar atención; identificación de señales de alarma, y conocimiento de derechos y deberes reproductivos referido por la paciente.

Segunda demora (barreras). Demora en identificar y acceder a un servicio de salud, dificultad para llegar a un acuerdo familiar, malas experiencias, recursos económicos deficientes, falta de disponibilidad transporte al momento de la emergencia obstétrica referida por la paciente.
Tercera demora (barreras). Demora en obtener el tratamiento adecuado y oportuno, aumento del tiempo de espera en la institución de segundo nivel, identificación de riesgos, falta de recursos médicos y materiales, medicamentos deficientes referido por la paciente.

Morbilidad materna. Complicaciones relacionadas de manera directa con el embarazo, parto o puerperio, registrados en el expediente y referido por la paciente.

Mortalidad materna. Defunción de la madre registrada en el expediente.

Mortalidad fetal. Defunción del feto o producto registrado en el expediente.

Nosotros hipotetizamos que las demoras se asocian con la morbilidad. Para el análisis estadístico se calcularon medias, porcentajes, razón de momios para estudiar las asociaciones de variables y x2 como prueba de hipótesis. Se utilizaron niveles de confianza del 95\% y diferencias en valores de $p<0.05$ se dieron como significativas.

\section{Resultados}

Durante el periodo de estudio se dio atención a un total de 42 embarazadas. Sin embargo, no se logró localizar a todas las pacientes, por lo que se realizaron un total de 35 entrevistas, cuyos expedientes físicos fueron revisados.

La media de edad de las embarazadas fue 27.6 años. Respecto a la escolaridad, el $17.1 \%$ careció de enseñanza básica. El $85.7 \%$ fueron amas de casa. El $28.5 \%$ no utilizó métodos de planificación familiar. El $45.1 \%$ tuvieron de 5 a 6 consultas prenatales.

De las 9 pacientes que no tuvieron ninguna morbilidad y que presentaron la primera demora las causas fueron $6(66.6 \%)$ falta de reconocimiento de datos de alarma, 1 (11.1\%) falta de acuerdo familiar para buscar ayuda, y 1 $(11.1 \%)$ se automedicó antes de acudir a recibir atención (Tabla 1). 


\begin{tabular}{|c|c|c|c|c|c|c|}
\hline \multicolumn{7}{|c|}{ Demora 1} \\
\hline & Total & $\%$ & $\begin{array}{l}\text { Con } \\
\text { morl }\end{array}$ & ilidad & $\begin{array}{l}\text { Sin } \\
\text { mo }\end{array}$ & ilidad \\
\hline Barrera & $\mathrm{N}^{\circ}$ & $\%$ & $\mathrm{~N}^{\circ}$ & $\%$ & $\mathrm{~N}^{\circ}$ & $\%$ \\
\hline $\begin{array}{ll}\text { Reconoce } & \\
\text { datos } & \text { de } \\
\text { alarma } & \\
\end{array}$ & 10 & 43.84 & 4 & 28.5 & 6 & 66.67 \\
\hline $\begin{array}{ll}\text { Acuerdo } & \text { en } \\
\text { búsqueda } & \text { de } \\
\text { ayuda } & \\
\end{array}$ & 4 & 17.39 & 3 & 21.4 & 1 & 11.11 \\
\hline $\begin{array}{ll}\text { Acude } & \text { a } \\
\text { sobadora }\end{array}$ & 1 & 4.35 & 1 & 7.14 & 0 & 0.00 \\
\hline $\begin{array}{l}\text { Le resta } \\
\text { importancia a } \\
\text { lo sentido }\end{array}$ & 3 & 13.04 & 2 & 14.2 & 1 & 11.11 \\
\hline $\begin{array}{l}\text { Se } \\
\text { automedica }\end{array}$ & 2 & 8.70 & 2 & 14.2 & 0 & 0.00 \\
\hline $\begin{array}{l}\text { Espera } \\
\text { remisión de } \\
\text { síntomas }\end{array}$ & 3 & 13.04 & 2 & 14.2 & 1 & 11.11 \\
\hline Total & 23 & 100.0 & 14 & 100.0 & 9 & 100.0 \\
\hline
\end{tabular}

Tabla 1 Barreras que causan demora 1, frecuencia y porcentaje en pacientes con y sin morbilidad

De las principales barreras encontradas que causan la segunda demora encontramos que $3(13.6 \%)$ no recibieron una hoja de referencia del centro de salud de primer nivel, ya que decidieron ir directo al segundo nivel, 9 (40.9\%) mencionó que a pesar de recibir hoja de referencia no se encontró ambulancia municipal de traslado disponible al momento de la urgencia, $4(18.1 \%)$ mencionaron que se retrasó en la búsqueda de atención ya que esperaron a algún familiar con posibilidad de traslado que las apoyara, 3 (13.3\%) argumentaron un elevado costo de traslado, y $3(13.6 \%)$ afirmaron que a pesar de tener ambulancia de traslado, hoja de referencia y todos los documentos pertinentes, las autoridades municipales se negaron a brindar apoyo si la paciente o familiares de la paciente no apoyaban al mismo partido político en gestión (Tabla 2).

\begin{tabular}{|l|r|r|r|r|r|r|}
\hline \multicolumn{7}{|c|}{ Demora 2 } \\
\hline & Total & $\%$ & \multicolumn{2}{l|}{$\begin{array}{l}\text { Con } \\
\text { morbilidad }\end{array}$} & \multicolumn{2}{l|}{$\begin{array}{l}\text { Sin } \\
\text { morbilidad }\end{array}$} \\
\hline Barrera & $\mathrm{N}^{\circ}$ & $\%$ & \multicolumn{1}{l|}{$\mathrm{N}^{\circ}$} & $\%$ & $\mathrm{~N}^{\circ}$ & $\%$ \\
\hline Sin referencia & 3 & 13.64 & 2 & 14.29 & 1 & 12.50 \\
\hline $\begin{array}{l}\text { No hubo } \\
\text { ambulancia }\end{array}$ & 9 & 40.91 & 6 & 42.86 & 3 & 37.50 \\
\hline $\begin{array}{l}\text { Esperó apoyo } \\
\text { de familiar }\end{array}$ & 4 & 18.18 & 3 & 21.43 & 1 & 12.50 \\
\hline $\begin{array}{l}\text { Costo elevado } \\
\text { de transporte }\end{array}$ & 3 & 13.64 & 2 & 14.29 & 1 & 12.50 \\
\hline $\begin{array}{l}\text { Transporte } \\
\text { condicionado } \\
\text { a partido } \\
\text { político }\end{array}$ & 3 & 13.64 & 1 & 7.14 & 2 & 25.00 \\
\hline Total & 22 & 100.0 & 14 & 100.0 & 8 & 100.0 \\
\hline NOTA: Se obtuvo un valor de ${ }^{2} 0.0459$ con una $\mathrm{p}=0.8303$ \\
\hline
\end{tabular}

Tabla 2 Barreras que causan demora 2, frecuencia y porcentaje en pacientes con y sin morbilidad
De las 5 pacientes que presentaron la tercera demora y que además cursaron con un embarazo normo evolutivo 1 (20\%) mencionó que tuvo un tiempo de espera largo sin conocer la causa del retraso, 1 (20\%) mencionó la gran demanda de pacientes como motivo del retraso en su atención, y 2 (40\%) no cumplían criterios de ingreso al momento de acudir por primera vez por lo que tuvieron que acudir varias veces en menos de 24 horas (Tabla 3 ).

\begin{tabular}{|l|r|r|r|r|r|r|}
\hline & Total & $\%$ & \multicolumn{2}{|c|}{$\begin{array}{l}\text { Con } \\
\text { morbilidad }\end{array}$} & \multicolumn{2}{l|}{$\begin{array}{l}\text { Sin } \\
\text { morbilidad }\end{array}$} \\
\hline Barrera & $\mathrm{N}^{\circ}$ & $\%$ & \multicolumn{1}{|c|}{$\mathrm{N}^{\circ}$} & $\%$ & $\mathrm{~N}^{\circ}$ & $\%$ \\
\hline Sin referencia & 4 & 19.05 & 3 & 18.75 & 1 & 20.00 \\
\hline $\begin{array}{l}\text { No hubo } \\
\text { ambulancia }\end{array}$ & 7 & 33.33 & 6 & 37.50 & 1 & 20.00 \\
\hline $\begin{array}{l}\text { Esperó apoyo } \\
\text { de familiar }\end{array}$ & 4 & 19.05 & 3 & 18.75 & 1 & 20.00 \\
\hline $\begin{array}{l}\text { Costo elevado } \\
\text { de transporte }\end{array}$ & 1 & 4.76 & 1 & 6.25 & 0 & 0.00 \\
\hline $\begin{array}{l}\text { Transporte } \\
\text { condicionado } \\
\text { a partido } \\
\text { político }\end{array}$ & 5 & 23.81 & 3 & 18.75 & 2 & 25.00 \\
\hline Total & 21 & 100.0 & 16 & 100.0 & 5 & 100.0 \\
\hline NOTA: Se obtuvo un valor de $\mathrm{x}^{2}$ de 4.17 con $\mathrm{p}=0.0411$ \\
\hline
\end{tabular}

Tabla 3 Barreras que causan demora 3, frecuencia y porcentaje en pacientes con y sin morbilidad

\section{Conclusiones}

Todas las pacientes presentaron por lo menos una demora, 25 (71.4\%) presentaron únicamente una demora, $7(20 \%)$ presentaron 2 demoras y 3 (8.5\%) presentaron las 3 demoras.

El $34.8 \%$ correspondieron a la primera demora $33.3 \%$ a la segunda demora y $31.8 \%$ la tercera demora.

Las principales barreras que causan demora fueron las siguientes: de la primera demora $28.5 \%$ fue por falta de reconocimiento de datos de alarma, $21.4 \%$ fue por falta de acuerdo familiar para la búsqueda de atención, $7.4 \%$ fue por que acude a un sobador o sobadora antes que al médico, $14.2 \%$ le resta importancia a la sintomatología, $14.2 \%$ se automedica antes de recibir atención, y $14.2 \%$ espera a que remitan los síntomas espontáneamente. 
De la segunda demora $13.6 \%$ no recibió una hoja de referencia del centro de salud de primer nivel ya que deciden ir directo a segundo nivel, $40.9 \%$ menciona que a pesar de recibir hoja de referencia no se encuentra ambulancia municipal de traslado disponible al momento de la urgencia, $18.1 \%$ menciona que se retrasa en la búsqueda de atención ya que esperan a algún familiar con posibilidad de traslado que las apoye, $13.3 \%$ argumentan un elevado costo de traslado, y $13.6 \%$ afirman que a pesar de tener ambulancia de traslado, hoja de referencia y todos los documentos pertinentes las autoridades municipales se niegan a brindar apoyo si la paciente o familiares de la paciente no apoyan al mismo partido político en gestión. De la tercera demora las principales barreras fueron $19.0 \%$ tiempo de espera larga para ser atendidas sin conocer el motivo del retraso, $33.3 \%$ gran demanda de pacientes en espera de ser atendidos, $19 \%$ ausencia de médico especialista al momento de la urgencia, $14.7 \%$ traslado del Hospital Materno a Hospital O’Horán por falta de equipo y recursos humanos necesarios para la atención de alguna complicación, $23.8 \%$ falta de criterios para ingreso lo que condicionó a algunas pacientes a acudir varias veces al hospital en menos de 24 horas.

Las principales causas de morbilidad encontramos infecciones genitourinarias en el embarazo presentada por $17.9 \%$ de las pacientes, $5.7 \%$ preeclampsia sin especificar, $5.7 \%$ preeclampsia moderada, $5.7 \%$ preeclampsia severa, $5.7 \%$ hipertensión gestacional, $7.6 \%$ con trabajo de parto complicado por sufrimiento fetal, de los cuales solamente uno resultó en óbito fetal. Del total de demoras, $68.18 \%$ de ellas se presentaron en pacientes que habían cursado con alguna morbilidad, $31.8 \%$ en pacientes con embarazos normoevolutivos. De las pacientes que tuvieron alguna morbilidad, $33.3 \%$ presentaron la primera demora, $31.1 \%$ la segunda demora y $35.5 \%$ la tercera demora. De las pacientes con embarazo normoevolutivo, sin ninguna morbildad $38.09 \%$ presentaron la primera demora, $38.09 \%$ la segunda demora, y $23.8 \%$ la tercera demora.

Por si sola únicamente la tercera demora presentó asociación a la morbilidad materna, con un valor de $\mathrm{x} 2$ de 4.17 con $\mathrm{p}=0.0411$. En conjunto las 3 demoras tienen un valor de $\mathrm{x} 2$ de 3.97 con $p=0.0462$, demostrando que de alguna forma las demoras se asocian con alguna morbilidad.

\section{Referencias}

Alkema L, Chou D, Hogan D, Zhang S, Moller AB, Gemmill A, et al. (2016). Global, regional, and national levels and trends in maternal mortality between 1990 and 2015, with scenariobased projections to 2030: a systematic analysis by the UN Maternal Mortality Estimation InterAgency Group. Lancet ;387(10017):462-74.

Bobadilla J L, Reyes F S\& Karchmer S. (1992) Magnitud y causas de la mortalidad materna en el Distrito Federal 1988-1989. Gac Med Mex; 132: 5-16.

Briones GJ, Reyes FA. Preeclampsia. México: Editorial Alfil, 2008.

Elú MC \& Santos-Pruneda E. (2004) Mortalidad materna: una tragedia evitable. Perinatol Reprod Hum;18(1):44-52

http://www.who.int/reproductivehealth/topics/ maternal_perinatal/nmconcept/en/

Estrategia Nacional para la Prevención del Embarazo en Adolescentes. Gobierno de la República. México. Retomado de: https://www.gob.mx/cms/uploads/attachment/fi le/55979/ENAPEA_0215.pdf

Faneite P. (2008) Mortalidad materna en la Región Bolivariana de Latinoamérica: área crítica. Rev Obstet Ginecol Venez. 68:18-24.

Faneite P, Rojas L \& Briceño G. (2006) Mortalidad materna, Análisis. Salus, Rev Fac de Ciencias Salud UC. 10:26-30.

Gamboa Montejano C \& Valdés Robledo S. Mortalidad materna en México. Marco conceptual, derecho comparado, políticas públicas, tratados internacionales, estadísticas y opiniones especializadas. Dirección General de Servicios de Documentación, Información y Análisis Dirección de Servicios de Investigación y Análisis Subdirección de Análisis de Política Interior. Septiembre 2015. Disponible en: http://www.diputados.gob.mx/sedia/sia/spi/SAP I-ISS-74-15.pdf

Informe semanal de vigilancia epidemiológica. Información hasta la semana 27 epidemiológica 01 del 2017. Sistema de notificación inmediata de muerte materna. Disponible en: https://www.gob.mx/cms/uploads/attachment/fi le/180156/MMAT_2017_SE01.pdf 
Karam C, Bustamante M, Campuzano M\& Pliego A. (2007) Aspectos sociales de la mortalidad materna: Estudiode casos en el Estado de México. Med Soc [Internet] [citado 15 Ene 2010]; 2: 205-211. En: www.medicinasocial.info.

Ministerio del Poder Popular para la Salud, Dirección General de Epidemiología. Manual de normas de vigilancia epidemiológica de muerte materna, Infantil y 1-4 años de edad (SIS-05. SIVIGILA 2008). Caracas; 2008.

Organización Panamericana de la Salud, Organización. mundial de la salud. [Internet] Clasificación estadística internacional de enfermedades y problemas relacionados con la salud; Washington 1995 [citado 14 May 2011]. En:

wWw.cemece.salud.gob.mx/fic/cie/index.html

Organización Mundial de la Salud.[Internet]maternal mortality in 2005. Estimates developed by WHO,UNICEF, UNFPA and The World Bank; Ginebra 2007[citado 25 Nov 2010. En: http://www.who.int/makingpregnancy safer/topics/maternal mortality/es/ Index.html.

Organización Panamericana de la Salud, Organización Mundial de la Salud. [Internet] Washington. Estrategia regional para la reducción de la mortalidad y morbilidad materna. 26a Conferencia Sanitaria Panamericana, 54a Sesión del Comité Regional; 2002 [citado 26 Mar 2010] En: www.paho.org

Organización Mundial de la Salud. Informe sobre la salud en el mundo. OMS; 2003. En:http://who.int/who/2003/en/chafter2-es.pdf. Patton GC, Coffey C, Sawyer SM, Viner RM, Haller DM, Bose K, Vos T, Ferguson J \& Mathers CD. (2009). Global patterns of mortality in young people: a systematic analysis of population health data. Lancet. 374:881-892.
Procedimiento: Referencia y Contrarreferencia de pacientes entre unidades médicas y servicios de atención primaria, hospitalaria y de especialidad Manual de Procedimientos para la Operación del Sistema de Referencia y Contrarreferencia de Pacientes en Unidades Médicas de Primero, Segundo y Tercer Nivel de Atención. Secretaría de Salud. Instituto de Salud del Estado de México. Enero 2010. Consultado el 5 de agosto de 2013 en: http:// www.edomex.gob.mx/legistelfon/doc/pdf/gct/2 012/abr113.PDF

Rodríguez-Angulo, Aguilar-Pech, MonteroCervantes, Hoil-Santos \& Andueza-Pech. (2012) Demoras en la atención de complicaciones maternas asociadas a fallecimientos en municipios del sur de Yucatán, México. Rev Biomed ; 23: 23-32

Rodríguez-Angulo, Aguilar-Pech, MonteroCervantes, Hoil-Santos \& Andueza-Pech. (2012) Demoras en la atención de complicaciones maternas asociadas a fallecimientos en municipios del sur de Yucatán, México. Rev Biomed. 23: 23-32

Rodríguez-Angulo E, Pech GA \& Vázquez RZ. (2018). Global risk of death due to delays in the care of pregnant women with obstetric complication in yucatan, Mexico. Obstet Gynecol Int J ;9(2):118-122. DOI: 10.15406/ogij.2018.09.00315

Romero, Mariana, Silvina Ramos, and Edgardo Ábalos. "Modelos de análisis de la morbimortalidad materna." Hoja informativa 3 (2010).

Rosado-Alcocer L, Rodríguez-Angulo E\& AnduezaPech G. (2008). Evaluación del modelo de los tres retrasos en las defunciones maternas en Mérida, Yucatán. En: Investigación y Salud 3. Ed. Universidad Autónoma de Yucatán. México. Pp 325-339

SSA, Dirección General de Información en Salud. In mujeres, Cálculos a partir de SSA. Sistema Nacional de Información en Salud, (SINAIS) [en línea]: Información dinámica: bases de datos en formato de cubo dinámico/Defunciones 1979-2016. CONAPO. Proyecciones de la Población de México 20052050 . 
Trinidad, C.S., Mateos, A.G., Moreno, F.S. \& Aguilar, A.R. (2015). Morbilidad y mortalidad materna y factores de riesgo asociados con una urgencia obstétrica. Ginecol Obstet Mex;83:96103.

Velasco-Murillo V. (2002) La mortalidad materna: un problema vigente hasta nuestros días. Rev Med IMSS;40(3):185- 186. 